\title{
Clinical features, severity, and outcomes of patients with COVID-19 infection: A follow-up study
}

\author{
Deldar Morad Abdulah, ${ }^{1}$ Hemin Khalid Saber ${ }^{2}$ \\ ${ }^{1}$ Community and Maternity Health Unit, College of Nursing, University of Duhok-Iraqi Kurdistan, Duhok \\ City; ${ }^{2}$ Department of Medicine, College of Medicine, Hawler Medical University, Iraqi Kurdistan, Erbil City, \\ Iraq
}

\begin{abstract}
Few epidemiological investigations explored disease outcomes in patients with COVID-19 in Iraqi Kurdistan. The association of clinical and epidemiological characteristics of COVID-19 patients with outcomes was examined in this study. Patients who were diagnosed with COVID-19 from a private clinic were followed up until recovery or death between $15^{\text {th }}$ August and $20^{\text {th }}$ October 2020. The mean age of the COVID-19 patients was $47.12(6-90$ years). Fatigue (76.15\%), myalgia (66.53\%), fever $(65.33 \%)$, cough $(62.53 \%)$, sweating $(58.52 \%)$, and headache $(56.11 \%)$ were the most prevalent symptoms. Most of the patients had mild sever-
\end{abstract}

Correspondence: Deldar Morad Abdulah, Community and Maternity Health Unit, College of Nursing, University of Duhok-Iraqi Kurdistan, Duhok city

Tel.: +9647507443319

E-mail: deldarmorad@gmail.com

Key words: COVID-19; disease severity, outcome assessment.

Acknowledgement: We would like to thank our deep thanks to the patients who assisted us in this study.

Contributions: DMA: review, statistical extractions, data analysis; HKS: assessment, diagnosis, data collection, analysis, and final approval

Conflict of interest: The Authors declare no conflict of interest.

Availability of data and materials: The raw data of this study are available upon request.

Ethics approval: The ethical approval was obtained from College of Medicine of Hawler Medical Sciences. The study is conformed with the Helsinki Declaration of 1964, as revised in 2013, concerning human and animal rights.

Informed consent: Not applicable.

Received for publication: 9 August 2021.

Revision received: 26 August 2021.

Accepted for publication: 10 September 2021.

This work is licensed under a Creative Commons Attribution NonCommercial 4.0 License (CC BY-NC 4.0).

(C) Copyright: the Author(s),2021

Licensee PAGEPress, Italy

Annals of Clinical and Biomedical Research 2021; 2:159

doi:10.4081/acbr.2021.159 ity $(350,70.14 \%)$ followed by moderate $(74,14.83 \%)$, severe $(46$, $9.22 \%)$, and critical $(29,5.81 \%)$. The case fatality rate (CFR) was $5.41 \%(\mathrm{n}=27)$ and was significantly increased with increasing severity; mild $(0.0 \%)$, moderate $(1.35 \%)$, severe $(10.87 \%)$, and critical $(72.41 \% ; \mathrm{P}<0.001)$. The patients with the critical and severe situations were significantly older; medians: 67 vs 55 years compared to those patients with moderate and mild situations; medians: 51 vs 45 years; $\mathrm{P}<0.001$. The patients who died were significantly older compared to recovered patients; medians: 65 vs 46 years; $\mathrm{P}<0.001$, respectively. The disease severity was the only factor to predict mortality in patients with COVID-19 disease. Concerning the severity, being older, having anorexia, fatigue, pleurisy, diarrhea, fever, sweating, shortness of breath, and being male were determined to be predictors of severity in COVID-19 patients. The incidence rate of severe/critical conditions was significantly increased with increasing age. The older age was determined to be a predictor for higher mortality.

\section{Introduction}

The novel coronavirus infection called COVID-19 was originated in China in 2019. ${ }^{1}$ The outbreak was spread to other countries and regions, including Iraqi Kurdistan. ${ }^{2}$ Worldwide, by 28 April 2021 the total reported confirmed and dead cases of COVID19 were 148,329,348 and 3,128,962 deaths, respectively. ${ }^{3}$ World Health Organization (WHO) has expressed deep concern about the spread and severity of this pandemic. ${ }^{4}$ The initial confirmed cases in Iraqi Kurdistan were among those individuals who returned from Iran in February 2020. Following that time, the Kurdistan Regional Government (KRG) released preventive measures for travelers and the general population. In this regard, public and private schools and universities were closed in response to this outbreak, and a curfew was imposed from March 13 until April 23, 2020. The individuals arriving from an epidemic area were quarantined for at least 14 days. $^{5}$

The viral agent of this disease is not completely understood yet. ${ }^{6}$ The common clinical characteristics of the COVID-19 disease are fever, shortness of breath, dry cough, and pneumonia. ${ }^{7}$ The disease has different severity from mild to critical illnesses. The severe form of the disease is developed in patients with some risk factors such as old age ( $\geq 65$ years), smokers, and coexisting medical conditions. ${ }^{8}$ The median incubation period of the COVID19 disease is 5-6 days; but, it takes up to 24 days. ${ }^{9}$ The disease transmission may occur in the early stages of the disease, but the infectivity period is not completely understood. ${ }^{10}$ The prevention of the disease is important to the overall control of the disease owing to great epidemiological concerns. The different degrees of abnormalities are found in the laboratory findings e.g. leukopenia, leukocytosis, lymphopenia etc. ${ }^{11}$

The diseases have various demographic and anthropometric 
characteristics in different geographic locations ${ }^{12}$ and these differences could affect clinical outcomes. We need to analyze data from different geographic areas to find out the specific treatment plan and optimize resource allocation. In this regard, we aimed to examine the association of clinical and epidemiological characteristics with outcomes in COVID-19 patients.

\section{Materials and Methods}

\section{Study design and patients}

In this follow-up study, the patients who were diagnosed with the COVID-19 disease by a pulmonologist from a private clinic were followed up until recovery or death. The patients had different severities, including mild, moderate, severe, and critical. The patients with severe and critical conditions were admitted to the hospital and were followed up from the hospitals. The patients were diagnosed at the private clinic in Erbil city in Iraqi Kurdistan between $15^{\text {th }}$ August and $20^{\text {nd }}$ October 2020. The pulmonologist diagnosed the COVID-19 cases based on the WHO interim guidance for COVID-1913 and local guidelines of the Ministry of Health. ${ }^{14}$ The patients were included in this study regardless of disease severity, coexisting medical conditions, and socio-demographic aspects. The severity of the disease was determined. ${ }^{14}$ The patients of both gender diagnosed with COVID-19 were eligible to include in this study.

\section{Diagnosis and Measures}

The general and clinical features of the patients were recorded in a pre-designed questionnaire. The diagnosis of the disease was made and the severity of the disease is rated as mild, moderate, severe, or critical. ${ }^{14}$

\section{Statistical analysis}

Incidence rates of symptoms, disease severity, outcomes of patients with COVID-19 were determined in number and percentage. The case fatality rate in patients with different severities and characteristics and association of outcomes with symptoms was examined in the Pearson Chi-squared test. The role of disease severity, symptoms, and general characteristics on outcomes of COVID-19 patients was examined in the binary logistic regression model. The statistical calculations were performed by JMP Pro 14.3.

Table 1. Case fatality rate in patients with different severities.

\begin{tabular}{lccc}
$\begin{array}{l}\text { Severity and outcomes } \\
(\mathrm{n=499)}\end{array}$ & \multicolumn{2}{c}{ Outcomes } & P \\
Disease severity & & & \\
$\quad$ Mild & $350(100)$ & $0(0.0)$ & $<0.01$ \\
Moderate & $73(98.65)$ & $1(1.35)$ & \\
$\quad$ Severe & $41(89.13)$ & $5(10.87)$ & \\
Critical & $8(27.59)$ & $21(72.41)$ & \\
Symptoms & & & 0.40 \\
Asymptomatic & $30(100)$ & $0(0.0)$ & \\
Symptomatic & $442(94.24)$ & $27(5.76)$ & \\
\hline Gender & & & 0.11 \\
$\quad$ Male & $234(92.86)$ & $18(7.14)$ & \\
Female & $238(96.36)$ & $9(3.64)$ & \\
\hline
\end{tabular}

Pearson chi-squared test was performed for statistical analyses.

\section{Ethical considerations}

The approval and guarantee to publish the data were obtained from the College of Medicine of Hawler Medical University in Iraqi Kurdistan.

\section{Results}

The mean age of the COVID-19 patients was 47.12 (Sta. deviation: 15.76) between 6 and 90 years and were males (252, $50.50 \%)$ and females $(247,49.50 \%)$, pregnant $(5,1.00 \%)$. Fatigue (76.15\%), myalgia $(66.53 \%)$, fever $(65.33 \%)$, cough $(62.53 \%)$, sweating $(58.52 \%)$, and headache $(56.11 \%)$ were the most prevalent symptoms in patients with COVID-19 (Figure 1).

Most of the COVID-19 patients had mild severity (350, $70.14 \%$ ) followed by moderate $(74,14.83 \%)$, severe $(46,9.22 \%)$, and critical $(29,5.81 \%)$. Accordingly, 27 patients (5.41\%) died and $472(94.59 \%)$ were recovered from the disease (Figure 2).

The study showed that CFR was significantly increased with increasing severity; mild $(0.0 \%)$, moderate $(1.35 \%)$, severe $(10.87 \%)$, and critical $(72.41 \% ; \mathrm{P}<0.001)$. But, the mortality was not associated with being symptomatic or asymptomatic $(\mathrm{P}=0.40)$ with no significant difference between male and female patients $(\mathrm{P}=0.11$, Table 1).

The patients with the critical and severe situation were significantly older; medians: 67 vs 55 years compared to those patients with moderate and mild situations; medians: 51 vs 45 years; $\mathrm{P}<0.001$. In addition, the patients who died of the COVID-19 were

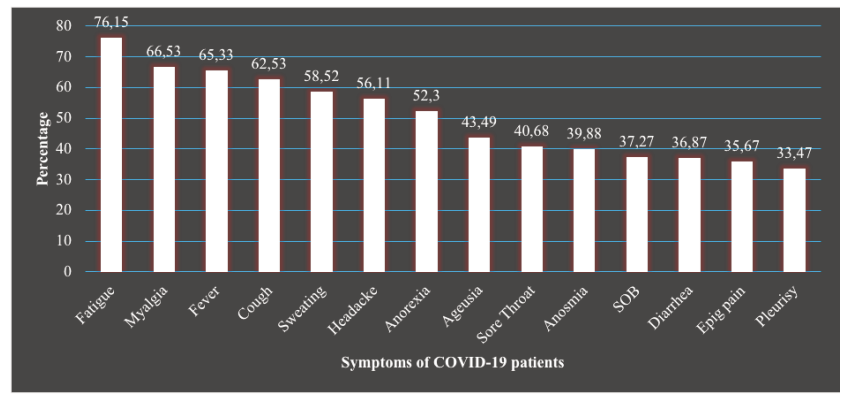

Figure 1. Incidence rates of symptoms in patients with COVID-19.

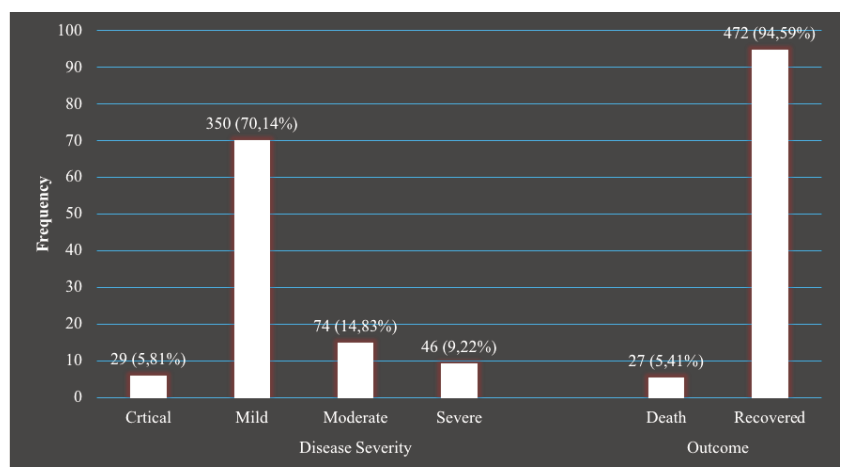

Figure 2. Disease severity and outcomes of patients with Covid-19. 
significantly older compared to recovered patients; medians: 65 vs. 46 years; $\mathrm{P}<0.001$, respectively (Figure 3 ).

The study found that the patients who had cough at presentation; $7.4 \%$ vs $2.1 \%$; $\mathrm{P}=0.012)$; shortness of breath (10.8\% vs $2.2 \%$; $\mathrm{P}<0.001)$; fatigue $(7.7 \%$ vs $2.9 \% ; \mathrm{P}=0.019)$, diarrhea $(8.2 \%$ vs $3.8 \% ; \mathrm{P}=0.038$ ) were more likely to die of the COVID-19.

The multivariate analysis through nominal logistic regression showed that disease severity is the only factor to predict mortality in patients with COVID-19 disease. The odds ratios of CFR were 446449807 ( 0 for moderate/mild); 10.44 (0.60-181.63; for severe/moderate; and 82.91 (9.17-749.55, for critical/severe). Concerning the severity, being older, having anorexia, fatigue, pleurisy, diarrhea, fever, sweating, shortness of breath, and being male were determined to be predictors of severity in COVID-19 patients (Table 2).

\section{Discussion}

The mean age of the COVID-19 patients was 47.12 between 6 and 90 years. Fatigue, myalgia, fever, cough, sweating, and headache were the most prevalent symptoms. The most prevalent severity of the disease was mild followed by oderate, severe, and critical. The CFR was significantly increased with increasing severity and age. The patients with the critical and severe situation were significantly older; medians: 67 vs 55 years compared to those patients with moderate and mild situations; medians: $51 \mathrm{vs}$ 45 years. The patients who had cough; shortness of breath; fatigue and diarrhea were more likely to die of the COVID-19. The disease severity was the only factor to predict the mortality in patients with COVID-19 disease.

The median age was lower compared to studies from Italy
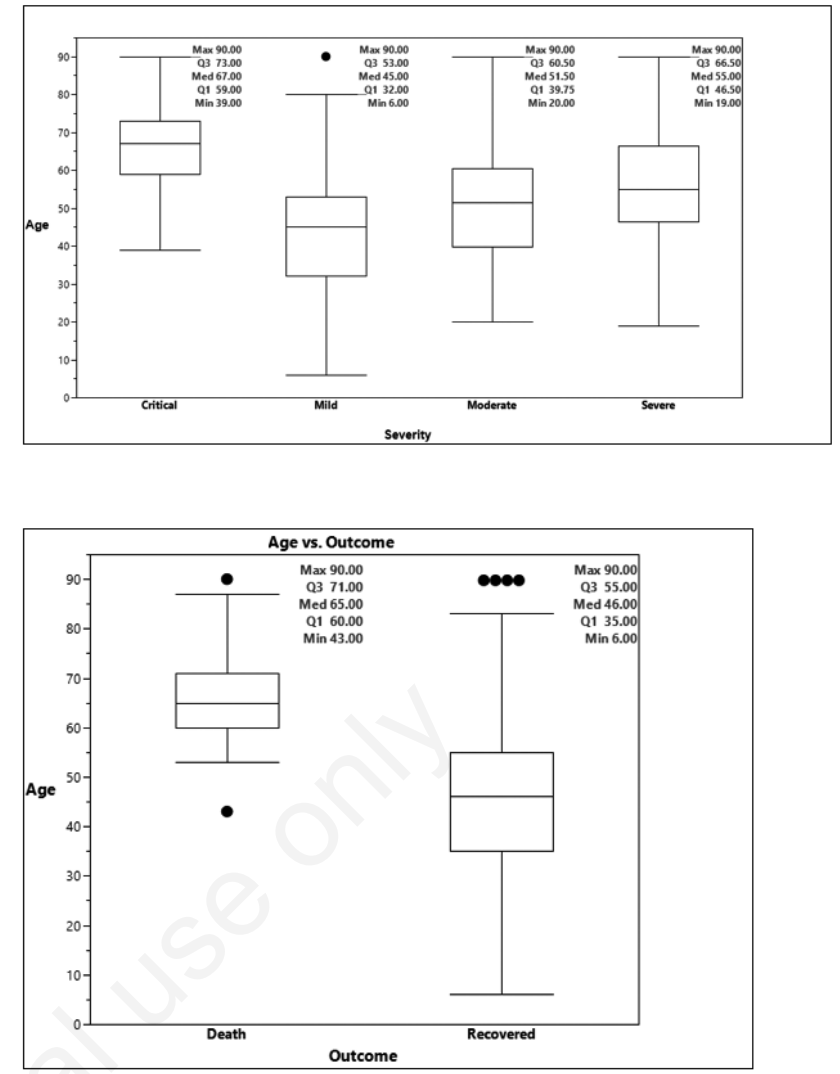

Figure 3. Comparisons of age in patients with different outcomes and severities.

Table 2. Role of disease severity, symptoms, and general characteristics on outcomes of COVID-19 patients

\begin{tabular}{|c|c|c|c|}
\hline Controlling factors $(\mathrm{n}=499)$ & $\begin{array}{c}\text { Dependent variable: Outcomes } \\
\text { OR }(95 \% \mathrm{CI})\end{array}$ & $\mathbf{P}$ & Dependent variable: Severity \\
\hline $\begin{array}{l}\text { Severity } \\
\text { Moderate/ Mild } \\
\text { Severe/ Moderate } \\
\text { Critical/ Severe }\end{array}$ & $\begin{array}{c}446449807 \text { (no lower-upper range) } \\
10.44(0.60-181.63 \\
82.91(9.17-749.55\end{array}$ & $<0.01$ & Outcome \\
\hline Anorexia & $8.68(0.86-87.55)$ & 0.05 & 0.011 \\
\hline Age & $1.06(0.99-1.12)$ & 0.06 & $<0.01$ \\
\hline Epigastric pain & $0.23(0.04-1.34)$ & 0.09 & 0.90 \\
\hline Fatigue & 483756045 (0 to .) & 0.14 & 0.01 \\
\hline Pleurisy & $0.26(0.03-1.97)$ & 0.17 & 0.01 \\
\hline Diarrhea & $3.10(0.48-19.94)$ & 0.21 & 0.01 \\
\hline Myalgia & $0.19(0.01-3.69)$ & 0.26 & 0.63 \\
\hline Ageusia & $0.34(0.04-3.10)$ & 0.33 & 0.86 \\
\hline Fever & $0.35(0.03-4.63)$ & 0.42 & 0.04 \\
\hline Cough & $0.39(0.02-8.41)$ & 0.55 & 0.17 \\
\hline Sore Throat & $0.52(0.06-4.67)$ & 0.56 & 0.42 \\
\hline Anosmia & $2.08(0.14-31.90)$ & 0.60 & 0.66 \\
\hline Sweating & $1.57(0.21-11.93)$ & 0.66 & 0.04 \\
\hline SOB & $1.53(0.17-14.00)$ & 0.71 & 0.01 \\
\hline Headache & $1.23(0.17-8.97)$ & 0.84 & 0.23 \\
\hline Sex (male/Female) & $0.89(0.16-4.87)$ & 0.89 & 0.01 \\
\hline
\end{tabular}

Nominal logistic regression was performed for statistical analysis. Point: The odds ratio was measured as per unit change in repressor. 
( 65.0 between 18 and $\geq 75$ years), ${ }^{15}$ China ( 47.0 between 0 and $\geq 65$ years), ${ }^{16}$ and (56.0 between 18 and 87 years), ${ }^{17}$ and the United States ( 63.0 between 0 and 107 years). ${ }^{18}$

In this study, the incidence rates of severe and critical COVID19 patients were $9.22 \%$ and $5.81 \%$, respectively. It was linked with the increasing age group. It is well documented in the literature that worse outcomes are associated with increasing age. ${ }^{16,19}$ Overall, increasing age is associated with impaired immunity and there is an increased prevalence of comorbid diseases such as diabetes mellitus and hypertension. The different rates of severe/critical conditions were reported in the literature, for instance, $15.74 \% .{ }^{16}$ The studies have reported that COVID-19 patients with severe/critical conditions are older with different median years, 7 years in China. ${ }^{16}$

This study reveals that $6.01 \%$ of the patients were asymptomatic upon visiting a medical doctor. Usually, few asymptomatic patients visit the hospitals for medical care. Fatigue, myalgia, fever, cough, sweating, and headache were the most prevalent symptoms. Concerning the severity, being older, having anorexia, fatigue, pleurisy, diarrhea, fever, sweating, shortness of breath, and being male were determined to be predictors of severity in COVID-19 patients. The patients who had cough; shortness of breath; fatigue, diarrhea were more likely to die of the COVID-19. The literature has reported that fever and cough on admission are the most prevalent symptoms in COVID-19 patients. Guan et al. ${ }^{16}$ reported that $43.8 \%$ and $88.7 \%$ had fever on admission and during hospitalization, respectively and $67.8 \%$ had cough. In addition, the patients with coexisting illnesses were more likely to have severe disease compared to non-severe patients, $38.7 \%$ vs $21.0 \%$, respectively. ${ }^{16}$ Similar findings were reported in other parts of the words in terms of symptoms. ${ }^{15,20}$ A meta-analysis confirmed that fever and cough are the most frequent symptoms. ${ }^{21}$ A meta-analysis of 148 articles of 24,410 adults with confirmed COVID-19 from 9 countries reported that fever (78\%), cough (57\%), and fatigue $(31 \%)$ are the most prevalent symptoms.

The CFR was $5.41 \%$ among 499 patients who were followed up and included in this study. The CFR rate was significantly increased with increasing age and disease severity. The CFRs are different based on the coexisting chronic conditions, clinical features, and epidemiological factors. A meta-analysis of 148 articles reported a $7 \%$ mortality rate. ${ }^{22}$ The early findings reported from China revealed that CFR is $1.4 \%$ in laboratory-confirmed COVID19 patients ${ }^{16}$ and $24.0 \%$ in Italy. ${ }^{15}$ The Italian study reported that patients aged older than 65 years, history of coronary artery disease, active cancer as independent factors related to increased mortality risk. Zhou et al. ${ }^{17}$ performed a multivariable regression and showed that in-hospital mortality is associated with older age, ddimer greater than $1 \mu \mathrm{g} / \mathrm{mL}$ on admission, and higher Sequential Organ Failure Assessment score.

The disease severity was the only predictor of mortality in COVID-19 patients. Old age was determined to be the predictor of severity in this study. Some other factors have been reported to be predictors of mortality. For example, Ciceri et al. ${ }^{15}$ reported that through multivariable analysis that older age, coronary artery disease, cancer, low lymphocyte count, and high RALE (Radiographic Assessment of Lung Edema) score as factors independently associated with an increased risk of mortality. Other investigations have reported that older age and the presence of comorbidities are associated with an increased mortality rate in COVID-19 patients..$^{17,20,23}$ We confirmed the previously published findings in patients from China, ${ }^{24}$ United States, ${ }^{18}$ and Italy. ${ }^{15}$ They reported that older age, coexisting medical conditions such as coronary artery disease, history of hypertension, diabetes, COPD, and chronic renal failure, and cancer are related to increased mortality. We back the effect of chronic diseases on increased mortality to their effect on immunity. ${ }^{25}$ The current findings give us the utmost importance to reduce the burden of the general health system, targeting the efforts for sufficient screening of the patients at risk. The role of age in immune system suppression must not be ignored in this disease. The countries with higher infection rates have an older population compared to countries with low infection rates, ${ }^{26}$ France and Italy compared to Iraq and Saudi Arabia, respectively. Older adults and patients with coexisting medical conditions are more likely to have a severe-even deadly-coronavirus infection. ${ }^{27}$ Therefore, the high infection and mortality rates in high-income countries could be due to aging and accordingly low immunity level. A recent study reported that the crude mortality rate has been increased by increasing consumption of sugarsweetened beverages and decreased by increasing fruit consumption and beans and legumes. ${ }^{28}$

Except for the insufficient age-related micronutrient, the nutritional status of the population has a role in the overall development of the SARS-CoV-2 infection, the clinical status, and outcomes. Therefore, the individuals need the maintenance of host macro-and micronutrient status to avoid the COVID-19 infection. ${ }^{29}$

Older individuals have less ability to respond to immune challenges and pathogens, antigens, and mitogens. ${ }^{30}$ Besides, the older persons have reduced the production of T cells in the involved thymus. Accordingly, this decreases the function of mature lymphocytes in secondary lymphoid tissues. ${ }^{31}$

\section{Implications and limitations}

The main strong point of this study is that we tried to include as much as possible the patients with sufficient information that allowed us to present a clearer picture of clinical features of the COVID-19 in this region. The predictors reported in this study could assist clinicians to determine at an early stage patients with COVID-19 with poor prognosis. However, the study was not exempt from the limitations. We could not record the radiological and laboratory-based information of this large study. Anyhow, we suggest that a large study of radiological and laboratory-based information be performed in a multi-center investigation.

\section{Conclusions}

This study showed that patients with COVID-19 have a low incidence rate of severe/critical status in Iraqi Kurdistan. The incidence rate of severe/critical conditions was significantly increased with increasing age. We found that older COVID-19 patients were more likely to have severe/critical status and a high rate of CFR. Old age was determined to be the only factor that predicted higher mortality.

\section{References}

1. Zhu N, Zhang D, Wang W, et al. A novel coronavirus from patients with pneumonia in China, 2019. N Engl J Med 2020;382:727-33.

2. Abdulah DM, Qazli SSA, Suleman SK. Response of the Public to Preventive Measures of COVID-19 in Iraqi Kurdistan. Disaster Med Public Health Prep 2020:1-9.

3. World Health Organization. WHO Coronavirus Disease (COVID-19) Dashboard: World Health Organization; 2020. 


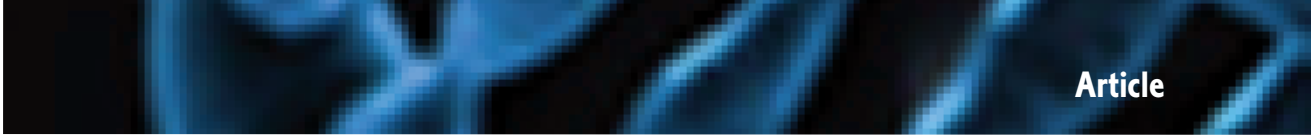

Accessed: 24 June 2021. Available from: https://covid19.who.int/

4. Nacoti M, Ciocca A, Giupponi A, et al. At the epicenter of the Covid-19 pandemic and humanitarian crises in italy: changing perspectives on preparation and mitigation. NEJM Catalyst Innovations in Care Delivery 2020;1.

5. (COVID-19), What the KRG is doing 2020. Available from: https://gov.krd/coronavirus-en/situation-update/

6. Cucinotta D, Vanelli M. WHO declares COVID-19 a pandemic. Acta Bio-medica: Atenei Parmensis 2020;91:157-60.

7. Adhikari SP, Meng S, Wu Y-J, et al. Epidemiology, causes, clinical manifestation and diagnosis, prevention and control of coronavirus disease (COVID-19) during the early outbreak period: a scoping review. Infect Dis Poverty 2020;9:1-12.

8. Centers for Disease Control and Prevention, 2021. Underlying medical conditions associated with high risk for severe COVID-19: Information for healthcare providers. Avaliable from: https://www.cdc. gov/coronavirus/2019-ncov/hcp/clinical-care/underlyingconditions.html

9. Kolifarhood G, Aghaali M, Mozafar Saadati H, et al. Epidemiological and clinical aspects of COVID-19; a narrative review. Arch Acad Emerg Med 2020;8:e41.

10. Zou L, Ruan F, Huang M, et al. SARS-CoV-2 viral load in upper respiratory specimens of infected patients. New Engl J Med 2020;382:1177-9.

11. Chen N, Zhou M, Dong X, et al. Epidemiological and clinical characteristics of 99 cases of 2019 novel coronavirus pneumonia in Wuhan, China: a descriptive study. The Lancet 2020;395:507-13.

12. Dulloo AG, Jacquet J, Solinas G, et al. Body composition phenotypes in pathways to obesity and the metabolic syndrome. Internat J Obesity 2010;34:S4-7.

13. World Health Organization. Global surveillance for COVID19 disease caused by human infection with the 2019 novel coronavirus, Interim guidance, 27 February 2020. WHO, 2020. Available from: https://apps.who.int/iris/handle/10665/331231

14. Merza MA, Al Mezori AAH, Mohammed HM, Abdulah DM. COVID-19 outbreak in Iraqi Kurdistan: The first report characterizing epidemiological, clinical, laboratory, and radiological findings of the disease. Diab Metabol Syndr 2020;14:54754.

15. Ciceri F, Castagna A, Rovere-Querini P, et al. Early predictors of clinical outcomes of COVID-19 outbreak in Milan, Italy. Clin Immunol 2020;217:108509.

16. Guan W-j, Ni Z-y, Hu Y, et al. Clinical characteristics of coronavirus disease 2019 in China. New Engl J Med 2020;382:1708-20.

17. Zhou F, Yu T, Du R, et al. Clinical course and risk factors for mortality of adult inpatients with COVID-19 in Wuhan, China: a retrospective cohort study. Lancet 2020;395:1054-62.

18. Richardson S, Hirsch JS, Narasimhan M, et al. Presenting characteristics, comorbidities, and outcomes among 5700 patients hospitalized with COVID-19 in the New York City area. JAMA 2020;323:2052-59.

19. Palaiodimos L, Kokkinidis DG, Li W, et al. Severe obesity, increasing age and male sex are independently associated with worse in-hospital outcomes, and higher in-hospital mortality, in a cohort of patients with COVID-19 in the Bronx, New York. Metabolism 2020;108:154262.

20. Wang D, Hu B, Hu C, et al. Clinical characteristics of 138 hospitalized patients with 2019 novel coronavirus-infected pneumonia in Wuhan, China. JAMA 2020;323:1061-9.

21. Sun P, Qie S, Liu Z, et al. Clinical characteristics of hospitalized patients with SARS-CoV-2 infection: a single arm metaanalysis. J Med Virol 2020;92:612-17.

22. Grant MC, Geoghegan L, Arbyn M, et al. The prevalence of symptoms in 24,410 adults infected by the novel coronavirus (SARS-CoV-2; COVID-19): A systematic review and metaanalysis of 148 studies from 9 countries. PLoS One 2020;15:e0234765.

23. Yang X, Yu Y, Xu J, et al. Clinical course and outcomes of critically ill patients with SARS-CoV-2 pneumonia in Wuhan, China: a single-centered, retrospective, observational study. Lancet Respir Med 2020;8:475-81.

24. Ganatra S, Hammond SP, Nohria A. The novel coronavirus disease (COVID-19) threat for patients with cardiovascular disease and cancer. JACC CardioOncol 2020;2:350-55.

25. Bagatini MD, Cardoso AM, Reschke CR, Carvalho FB. Immune system and chronic diseases 2018. J Immunol Res 2018;2018:1-2.

26. Ofori-Asenso R, Chin KL, Curtis AJ, et al. Recent patterns of multimorbidity among older adults in high-income countries. Population Health Manag 2019;22:127-37.

27. World Health Organization. Coronavirus disease 2019 (COVID-19): situation report, 72. WHO, 2020. Available from: https://covid19.who.int/

28. Abdulah DM, Hassan A. Relation of dietary factors with infection and mortality rates of COVID-19 across the world. J Nutrition Health Aging 2020:1-8.

29. Gasmi A, Noor S, Tippairote T, et al. Individual risk management strategy and potential therapeutic options for the COVID-19 pandemic. Clinical Immunol 2020:108409.

30. Chandra RK. Nutrition and the immune system from birth to old age. Eur J Clinical Nutrition 2002;56:S73-6.

31. Montecino-Rodriguez E, Berent-Maoz B, Dorshkind K. Causes, consequences, and reversal of immune system aging. J Clinical Investig 2013;123:958-65. 